\title{
BMJ Open Interventions to increase access to or uptake of physical health screening in people with severe mental illness: a realist review
}

\author{
Frédérique Lamontagne-Godwin, ${ }^{1}$ Caroline Burgess, ${ }^{2}$ Sarah Clement, ${ }^{3}$ \\ Melanie Gasston-Hales, ${ }^{4}$ Carolynn Greene, ${ }^{4}$ Anne Manyande, ${ }^{1}$ Deborah Taylor, ${ }^{4}$ \\ Paul Walters, ${ }^{5}$ Elizabeth Barley ${ }^{1}$
}

To cite: Lamontagne-Godwin F, Burgess C, Clement S, et al. Interventions to increase access to or uptake of physical health screening in people with severe mental illness: a realist review. BMJ Open 2018;8:e019412. doi:10.1136/ bmjopen-2017-019412

- Prepublication history and additional material for this paper are available online. To view these files, please visit the journal online (http://dx.doi org/10.1136/bmjopen-2017019412).

Received 31 August 2017 Revised 15 November 2017 Accepted 7 December 2017

Check for updates

${ }^{1}$ School of Human and Social Sciences, University of West London, London, UK

${ }^{2}$ Primary Care and Public Health Sciences, King's College London, London, UK

${ }^{3}$ Freelance Researcher, London, UK

${ }^{4}$ College of Nursing, Midwifery and Healthcare, University of West London, London, UK ${ }^{5}$ Department of Research and Development, Dorset HealthCare University NHS Foundation Trust, Bournemouth, UK

Correspondence to Frédérique Lamontagne-Godwin; frederique.lamontagnegodwin@uwl.ac.uk

\section{ABSTRACT}

Objectives To identify and evaluate interventions aimed at increasing uptake of, or access to, physical health screening by adults with severe mental illness; to examine why interventions might work.

Design Realist review.

Setting Primary, secondary and tertiary care. Results A systematic search identified 1448 studies, of which 22 met the inclusion criteria. Studies were from Australia ( $n=3)$, Canada $(n=1)$, Hong Kong $(n=1)$, UK $(n=11)$ and USA $(n=6)$. The studies focused on breast cancer screening, infection preventive services and metabolic syndrome (MS) screening by targeting MSrelated risk factors. The interventions could be divided into those focusing on (1) health service delivery changes (12 studies), using quality improvement, randomised controlled trial, cluster randomised feasibility trial, retrospective audit, cross-sectional study and satisfaction survey designs and (2) tests of tools designed to facilitate screening (10 studies) using consecutive case series, quality improvement, retrospective evaluation and pre-post audit study designs. All studies reported improved uptake of screening, or that patients had received screening they would not have had without the intervention. No estimation of overall effect size was possible due to heterogeneity in study design and quality. The following factors may contribute to intervention success: staff and stakeholder involvement in screening, staff flexibility when taking physical measurements (eg, using adapted equipment), strong links with primary care and having a pharmacist on the ward.

Conclusions A range of interventions may be effective, but better quality research is needed to determine any effect size. Researchers should consider how interventions may work when designing and testing them in order to target better the specific needs of this population in the most appropriate setting. Behaviour-change interventions to reduce identified barriers of patient and health professional resistance to screening this population are required. Resource constraints, clarity over professional roles and better coordination with primary care need to be addressed.
Strengths and limitations of this study

- In line with the realist review methodology, a broad and inclusive study identification process was used, which was adapted iteratively to compensate for the inconsistency around how terms such as 'screening' and 'monitoring' are used.

- A realist review explores why interventions might work in a particular setting, however studies provide limited evidence for this, so transferability of knowledge to other settings is limited.

- A realist review is often selected to understand complex interventions, however behaviour change theory was not included in almost any intervention design, making it impossible to discern which 'active ingredients' are at work to produce the results.

\section{INTRODUCTION}

People with severe mental illness (SMI), such as schizophrenia or bipolar disorder, have been found to have a twofold to threefold increased risk of premature mortality. ${ }^{1} \mathrm{~A}$ reduction in life expectancy of 10-20 years has been reported. ${ }^{23} \mathrm{~A}$ significant cause of this disparity is attributed to preventable and treatable long-term physical health conditions, with cardiovascular disease and cancer as the first and second leading causes of death, respectively, in this group. ${ }^{24}$ Cancer mortality in people with SMI is more likely than in the general population, ${ }^{5}$ though the incidence of disease is similar for both groups. ${ }^{6}$ One factor which contributes to inequality in survival rates is access to cancer screening. ${ }^{7}$ The reported $30 \%$ higher case fatality rate from cancer may partly be due to those with SMI being more likely to present with metastases at diagnosis. ${ }^{5}$

Other physical health conditions found to be more prevalent in people with SMI include type 2 diabetes and metabolic 
syndrome (MS), ${ }^{8}$ tuberculosis, HIV, osteoporosis, poor dentition, impaired lung function, sexual dysfunction and obstetrical complications. ${ }^{2}$ MS is defined by the $\mathrm{WHO}^{10}$ as glucose intolerance, diabetes mellitus and/ or insulin resistance, with two or more of the following: central obesity (waist-to-hip ratio: $\geq 0.90$ in men and $\geq 0.85$ in women and/or body mass index (BMI) $>30 \mathrm{~kg} / \mathrm{m}^{2}$ ), raised arterial pressure $(\geq 140 / 90 \mathrm{~mm} \mathrm{Hg})$, microalbuminuria $(\geq 20 \mu \mathrm{g} / \mathrm{min}$ or albumin:creatinine ratio $\geq 30$ $\mu \mathrm{g} / \mathrm{mg})$ and raised plasma triglyceride $(\geq 150 \mathrm{mg} / \mathrm{dL}$ and/or low high-density lipoprotein cholesterol (HDL-C; $<35 \mathrm{mg} / \mathrm{dL}$ in men and $<39 \mathrm{mg} / \mathrm{dL}$ in women)).

Health screening facilitates early detection and treatment for many of these conditions, though rates of screening in people with SMI may be reduced compared with the general population. A UK survey ${ }^{11}$ found that only $33 \%$ of people with schizophrenia had received adequate cardiovascular disease screening in the previous 12 months and, internationally, uptake of breast, cervical and bowel cancer screening has been found to be lower among people with SMI. ${ }^{612-15}$ Effective interventions for increasing access to, or uptake of, screening for a range of conditions in the general population ${ }^{16}$ exist. In relation to cancer screening, a Cochrane review ${ }^{17}$ found that no intervention to promote uptake has been tested in people with SMI. This is important since qualitative work ${ }^{18}$ indicates there are specific barriers to cancer screening uptake in people with SMI and that interventions effective in the general population may not be effective in the SMI population. Furthermore, barriers to screening uptake or access may vary for different types of screening, at different stages of the screening process and between individuals. $^{18}$

Realist review methodology ${ }^{19}$ has been devised to enable synthesis of diverse literature in order to explore what works for whom (as in traditional systematic reviews) and why an intervention may work. ${ }^{20}$

By drawing on this methodology, our objectives were to identify and evaluate interventions which may increase uptake of, or access to, any kind of physical health screening by adults with SMI, and to determine what works for whom in what setting and why. The review is described in accordance with the Realist And Meta-narrative Evidence Syntheses: Evolving Standards (RAMESES) reporting guidance for realist reviews. ${ }^{21}$

\section{METHODS}

\section{Study selection}

\section{Inclusion and exclusion criteria}

Studies of any intervention to promote access to, or uptake of, screening or monitoring for any physical health condition where participants were aged 18 years and over with a diagnosis of SMI (psychosis or bipolar disorder however diagnosed) were eligible. Uptake of screening was the main outcome of interest. Patient-related outcomes were not an inclusion criteria, but were included in the online supplementary tables following the review of the studies, to provide important additional information and give a rounded picture of the effectiveness of the interventions. The UK National Screening Committee defines screening as a "public health service in which members of a defined population... are asked a question or offered a test, to identify those individuals who are more likely to be helped than harmed by further tests or treatment to reduce the risk of a disease or its complications'. ${ }^{22}$ 'Monitoring' was defined in a Cochrane ${ }^{23}$ review as a means 'to obtain information which can then be acted on to treat or prevent a physical health problem'. We included any intervention described as promoting either screening or monitoring; for clarity the term 'screening' is used throughout. Only studies reported in English were included.

In line with the realist approach to literature synthesis, ${ }^{19}$ an inclusive approach was taken and intervention studies of any design were eligible as long as the full text was published in a peer-reviewed journal. We also excluded intervention studies to improve physical health in people with SMI which may involve screening, but where uptake or access to screening was not a main outcome and service evaluations or audits which considered screening, but did not test any intervention.

\section{Search strategy}

The protocol is published on the International Prospective Register of Systematic Reviews (PROSPERO) database. ${ }^{24}$ The search strategy (online supplementary appendix 1) was informed by published, related systematic reviews ${ }^{81720}$ and was checked by a specialist health librarian at the University of West London (Marc Forster, PhD). Searching was conducted in December 2016.

\section{Data sources}

MEDLINE, Embase, Cumulative Index to Nursing and Allied Health Literature, PsychINFO, Cochrane Database of Systematic Reviews, Database of Abstracts of Reviews of Effectiveness. Reference chaining of identified studies was also conducted. No date restrictions were applied.

\section{Search results}

The initial electronic search identified 1872 potentially relevant publications; six others were identified through reference chaining. Titles and abstracts were screened independently by two team members (EB and AM). Thirty-three full texts were retrieved and screened by three team members (EB, AM, DT). Among the thirty-three full texts was a recent systematic review of studies of 'Strategies to implement physical health monitoring in people affected by severe mental illness ${ }^{25}$ which included 14 studies. Though the focus of this review was slightly different from the current, it contained one study which we had included. ${ }^{26}$ It also included two studies which we had excluded: one $^{27}$ was not an intervention study, the other tested the validity of a health monitoring tool. ${ }^{28}$ This led to a team discussion whereby it was decided that studies of interventions, such as health monitoring tools, 


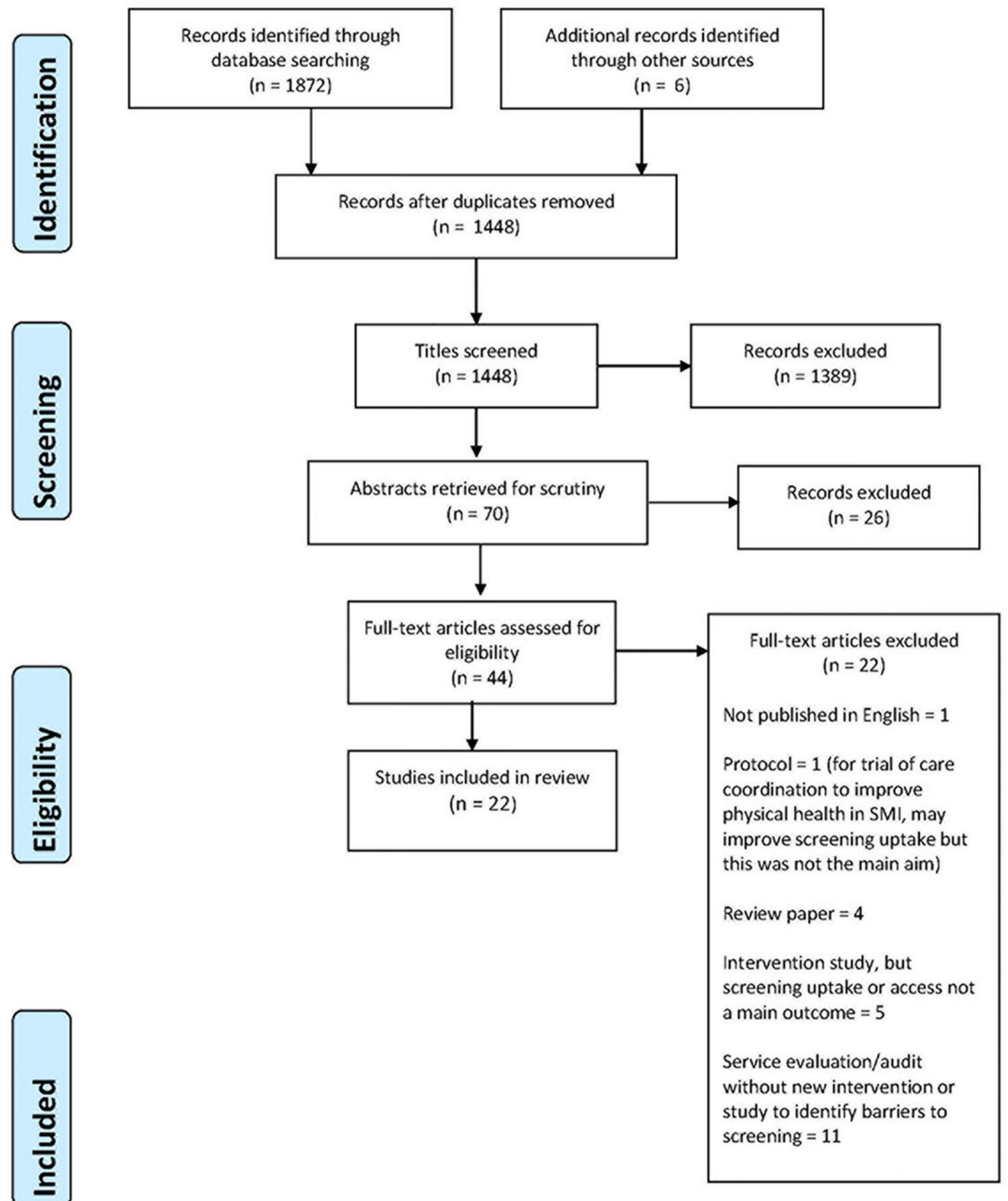

Figure 1 Preferred Reporting Items for Systematic Reviews and Meta-Analyses flow diagram of intervention studies included in, and excluded from, this review. SMI, severe mental illness.

were relevant to our review question. The rationale being that, although the main aim of these interventions was to improve the quality of screening (eg, more health indicators measured) and ongoing monitoring, this often resulted in increased uptake. We rescreened our identified studies and those included in this review. ${ }^{25}$ Fortyfour studies were identified as potentially relevant and were screened by two reviewers. Twenty-two of these did not meet the inclusion criteria, a total of 22 studies were included. The screening and study selection processes are detailed in figure 1 .

\section{Data extraction}

Each reviewer independently extracted information from up to five articles, with one author (EB) reviewing all studies. Data were extracted regarding study authors, geographical location and setting, year of publication, participant characteristics, features of the intervention, target of screening, outcome measures, study design and limitations.

\section{Approach to synthesis}

Similarities in intervention approach were identified and summarised across studies. Exploration of how and why different approaches might have worked was undertaken by searching for themes across studies, paying particular attention to disconfirming evidence. As there was considerable between-study variation in outcome measures, meta-analysis was not possible.

\section{RESULTS}

\section{Study characteristics}

Study characteristics are detailed in online supplementary tables 1 and 2. Two studies ${ }^{29} 30$ included breast cancer screening, one ${ }^{30}$ considered infection preventive services and 21 studies considered MS screening by targeting MS-related risk factors (cholesterol/ sugar), ${ }^{26} 28$ 31-48 blood pressure (BP) ${ }^{26}{ }^{28-31}{ }^{33-46}$ and BMI. ${ }^{26} 28$ 30-46 48 Two studies involved national screening programmes $^{29} 30$ and 20 studies developed 'in-house' screening. ${ }^{26} \quad 28 \quad 31-48$ Study populations included 
participants with schizophrenia, ${ }^{28-3133-3537384044-47}$ bipolar disorder, ${ }^{28-3133353844-47}$ schizoaffective disorder, ${ }^{282931333544-47}$ other psychotic disorders 88303435384546 and other mental health disorders. ${ }^{29-31} 3338$ 44-46 Some studies did not specify the SMI ${ }^{26} 394243$ while other studies included patients with SMI on antipsychotics ${ }^{32} 36414748$ with no breakdown by condition. Some of the participants in a few of the included studies had mental health disorders other than SMI. In those studies, there was a minimum of $45 \%$ participants who had either a psychosis or bipolar disorder diagnosis.

A range of study designs was employed (pre-post audit $\mathrm{n}=9$, consecutive prospective case series design $\mathrm{n}=1$, repeat audit $\mathrm{n}=1$, cross-sectional study $\mathrm{n}=1$, Quality Improvement (QI) $\mathrm{n}=4$, retrospective audit $\mathrm{n}=4$, randomised controlled trial $n=1$, cluster randomised feasibility trial $\mathrm{n}=1$ ). Study quality of randomised trials ${ }^{45}{ }^{46}$ was assessed using the Cochrane tool ${ }^{49}$ for assessing risk of bias. No similar 'gold standard' tool exists which could be used across the other study designs, so we assessed each study informed by a simple checklist based on the STrengthening the Reporting of OBservational studies in Epidemiology ${ }^{50}$ statement and a recent review of tools to assess bias in observational studies. ${ }^{51}$ Each study was rated independently by two reviewers with discrepancies resolved by discussion. Flaws relating to the reliability of findings or the generalisability of results were highlighted in all studies (online supplementary tables 1 and 2); these data suggest that findings concerning the size of effect should be considered with caution.

\section{Review outcomes}

Interventions to increase uptake of screening are defined as interventions which support health professionals to screen for physical health conditions. ${ }^{28}{ }^{32-48}$ Interventions to increase access to screening are defined as interventions (targeted at health professionals or health service delivery) to increase patient/client access to screening. ${ }^{26}$ 29-31

\section{Intervention effects}

Overall, improvements in rates of screening were reported in all studies following intervention (online supplementary tables 1-2). This appeared to be independent of screening type or study design. We identified an evidence gap as only three studies ${ }^{31} 3248$ reported whether the results of screening were acted on, for instance through referral or clinical intervention.

\section{Intervention type}

Studies broadly either tested a new tool to facilitate screening for health professionals ${ }^{28} 32 \quad 33 \quad 35-3947$ (online supplementary table 1) or made complex health services delivery changes ${ }^{26}$ 29-31 40-46 48 (online supplementary table 2 ). One intervention ${ }^{26}$ primarily targeted service users, though in other studies ${ }^{29} 4044$ service user-focused interventions were included, for instance targeted education, ${ }^{33} 40$ self-management support ${ }^{44}$ or support to attend screening. ${ }^{29}$ Most studies included staff education $^{384043454648}$ and training ${ }^{2833353942}$ as part of the intervention.

The data-collection tools tested in online supplementary table 1 were designed to gather information required to improve MS screening ${ }^{32} 333847$ or physical health monitoring. ${ }^{28}$ 34-37 39 MS monitoring was evaluated using the following measurements: BP, smoking status, waist circumference (WC), fasting blood glucose (BG), BMI, triglycerides and HDL-C. These measures were based on the following clinical guidelines: National Institute for health and Care Excellence 2631 35-37 39-41; Maudsley Prescribing Guidelines $^{34} 37$ 40-42; US Preventive Services Task Force ${ }^{3044}$; National Heart, Lung and Blood Institute ${ }^{33}$; American Diabetes Association ${ }^{32-34} 38$ 47 ; Early Psychosis Prevention and Intervention Centre ${ }^{43}$; Psychotropic Therapeutic Guidelines ${ }^{48}$ and the American Psychiatric Association Practice. ${ }^{28} 3538$ Most interventions were multifaceted, so will appear in more than one cluster. As no studies were rated of good or moderate quality, it was difficult to assess whether findings of improvements in rates of screening are valid. The size of effect was not reported for any study.

\section{Screening template}

Eight studies ${ }^{2832-3436-39}$ evaluated the effectiveness of using a screening tool to increase uptake and raise staff awareness of physical health screening. Barriers to successful intervention implementation included 'social desirability bias,28 36 (patients self-report their health behaviour in an overly positive picture in an effort to please their key workers); low uptake of physical measurements (eg, $\mathrm{WC}^{32}{ }^{37} \mathrm{BP}^{38}$ fasting $\mathrm{BG}^{33}{ }^{37}$ ) and of data on sensitive topics $^{36}$; difficulty to capture monitoring results onto the tool $^{28} 3238$ 39; difficulty in obtaining equipment ${ }^{37} 38$ and accessing laboratory services ${ }^{33}$; lack of integration with primary care for treatment or referral ${ }^{333738}$; appointment non-adherence ${ }^{32}{ }^{33}$; lack of expertise in mental health professionals to interpret physical health results ${ }^{34}{ }^{37}$; workload issues ${ }^{32}{ }^{33}{ }^{38}$; staff reluctant to see MS screening as their responsibility. ${ }^{32-34}$ Authors across studies identified the following facilitators: investment of staff in physical health monitoring ${ }^{33} 36379$ and staff flexibility by using alternative equipment and tests. ${ }^{33} 39$

\section{Staff education and training}

Five studies ${ }^{28} 33353839$ included staff training as a component of the intervention. No author described the content or format of education interventions in detail. Barriers to successful intervention implementation included workload issues ${ }^{3335} 38$; lack of training to spot 'social desirability bias, ${ }^{28}$; lack of training in mean WC measurement. ${ }^{28}$ The following facilitators were identified: 'booster' education and team meetings ${ }^{33}$ and investment of staff in physical health monitoring. ${ }^{33} 39$ 


\section{Computer or paper prompt for staff}

Four studies $^{34} 373947$ tested a computer-based or paperbased prompt to support clinicians to monitor and screen physical health indicators. Barriers to the successful implementation of the intervention included technical constraints in terms of collecting measurement results ${ }^{394}$; low uptake of physical measurements (eg, $\mathrm{WC}^{37}$ and fasting $\mathrm{BG}^{3747}$ ); lack of expertise from mental health professionals to interpret physical health results ${ }^{34}{ }^{37}$; unclear communication channel between primary and secondary care ${ }^{34} 37$ and limited access to equipment and resources. ${ }^{3437}$ Having a clinical psychiatric pharmacist on the ward to remind clinicians to request investigations, such as blood tests when appropriate, and to provide the relevant guidelines and precautions when initiating hypolipidaemic medication, was a facilitator to the successful implementation of the intervention in two studies. ${ }^{3747}$

Online supplementary table 2 describes 12 studies which tested interventions that delivered change in a health service setting. Most interventions were multifaceted so appear in more than one cluster. All studies targeted adults, though in one study ${ }^{42}$ eligible participants were 14-35 years old. Studies took place in a clozapine clinic, ${ }^{48}$ Early Intervention in Psychosis Services, ${ }^{41-43}$ Community Mental Health Team (CMHT), ${ }^{31} 4445$ community drop-in centre ${ }^{29}$ and primary care. ${ }^{26}{ }^{30}$ Interventions were focused on metabolic/cardiovascular screening for all studies, except one ${ }^{29}$ which was designed to increase rates of mammography uptake. One study ${ }^{30}$ monitored uptake of national cancer screening services and metabolic screening. The size of effect was not reported for any study. All studies reported suboptimal screening and monitoring at baseline, with improved levels postintervention. However, limited evidence of actions, such as referral or intervention, occurring as a result of these improvements was reported.

\section{Staff education and training}

Six studies ${ }^{404243454648}$ provided some kind of staff (working in primary and secondary care) and patient education. All studies described increased rates of monitoring following the interventions. One study ${ }^{45}$ was rated as good quality, so generalisation of results is limited. Barriers to the successful implementation of the intervention included staff time constraints ${ }^{40} 48$; poor communication across the primary and secondary care interface $e^{43} 45$ and lack of clarity over scope of practice ${ }^{48}$; patient resistance to undergo physical measurements ${ }^{42}{ }^{46}$; staff resistance to change ${ }^{40456}$ and staff turnover. ${ }^{43} 46$ Facilitators included team ownership and team 'champions' to encourage screening ${ }^{40} 42$ and high visibility/structure around monitoring and better liaison with primary care. ${ }^{42} 48$

\section{Invitation letter to physical health screening}

Three studies ${ }^{26} 4042$ used an invitation letter from primary care to encourage patients to attend screening as part of a physical health check-up. All studies described increased rates of monitoring following the interventions. Barriers to the successful implementation of the intervention included patient resistance to undergo physical measurements ${ }^{42}$ and staff resistance to change. ${ }^{40}$ Facilitators included team ${ }^{40} 42$ ownership and team 'champions' to encourage screening; getting stakeholders involved ${ }^{40} 42$ and living in a suburban ${ }^{26}$ (rather than urban) area.

\section{Improving access to monitoring resources}

Four studies ${ }^{41} 434648$ tested interventions developed to improve the collection of physical health data to increase screening. Barriers to the successful implementation of the intervention included patient resistance and lack of motivation in the screening process ${ }^{41}{ }^{46}$; inadequate links with primary care ${ }^{41}{ }^{43}$; no clarity about who takes responsibility for screening ${ }^{41}{ }^{48}$; staff turnover ${ }^{43}{ }^{46}$; staff not perceiving physical health screening as a priority ${ }^{4146}$; time and resource (screening equipment) constraints ${ }^{41}{ }^{48}$; poor recording and knowledge of screening guidelines and tests. ${ }^{41}{ }^{48}$ Facilitators included high visibility and structure around monitoring ${ }^{48}$ and having a key worker system with key worker's duties involving screening. ${ }^{41}$

\section{Integrating care across health settings}

Seven studies ${ }^{29-31} 40424445$ were developed to evaluate and reduce the fragmentation of care between different care providers. New clinics to improve physical healthcare were set up and evaluated, ${ }^{29-31}$ two trials ${ }^{44}$ evaluated nurse-led care management and two studies audited improvement in awareness ${ }^{42}$ and communication ${ }^{40}$ within the multidisciplinary care coordination team. All reported improvement in physical health monitoring or uptake of screening tests postintervention. Two studies ${ }^{445}$ were rated as good quality. One study ${ }^{44}$ reported improvement in cardiovascular disease risk among intervention subjects of an effect size comparable with that seen in underserved populations without mental illness. Barriers to the successful implementation of the intervention included lack of coordination across the primary and secondary care interface ${ }^{3045}$; patient reluctance to attend appointment/undergo screening ${ }^{29}{ }^{42}$; staff resistance to change $^{4045}$ and lack of a prescribing provider. ${ }^{44}$ Facilitators included team investment in screening procedure and stakeholder involvement ${ }^{29} 314042$; psychosocial support and trust between patients and staff to help them obtain screening ${ }^{294}$ and availability of primary and specialist care. $^{29} 304245$

\section{Staff accompaniment to appointments}

Four studies ${ }^{29} 334044$ included accompaniment of service users to appointments as part of their intervention. This type of intervention addresses potential difficulties in locating and visiting unfamiliar places which has been reported as a barrier to cancer screening uptake by some service users. ${ }^{18}$ One study ${ }^{33}$ from online supplementary table 1 was added to this cluster as it included staff accompaniment to screening. Barriers to the successful implementation of the intervention included staff workload issues ${ }^{3340}$; difficulty to engage staff ${ }^{3340}$; patient reluctance to undergo 
screening $^{29} 33$ and difficulty to obtain an appointment/ appointment non-adherence. ${ }^{29}{ }^{33}$ Facilitators included staff feeling invested/having a sense of ownership with regard to physical health screening ${ }^{29}{ }^{33}$; ; having access to primary care/in-home phlebotomy services ${ }^{29} 3344$ and trust between clients and staff. ${ }^{29} 33$

\section{DISCUSSION}

\section{Statement of principal findings}

A large international body of work was identified with diversity in the number of physical health conditions and clinical settings. Challenges to increase uptake of physical health screening and monitoring in people with SMI was not unique to a particular country, setting or health service configuration. The studies illustrate that people with SMI come into contact with a number of different health services. Two tools to facilitate screening ${ }^{35}{ }^{39}$ and two health service delivery change ${ }^{2640}$ interventions were delivered in primary care. The remaining took place in inpatient and outpatient mental health services. Mental health staff performed 'in-house' screening, ${ }^{28} 313637434648$ ordered screening tests ${ }^{30-34} 3841-434547$ or acted as a broker between the patient and screening service. ${ }^{29} 334144$ Overall, there appears to be no strong evidence as to whether an intervention to increase uptake of screening would be better suited in primary or secondary care. Performing 'in-house' screening in mental health services rather than in a primary care context warrants further research, including what training and equipment this requires. In addition, mechanisms to establish and maintain strong links between primary care/screening clinics and mental health services to ensure patients attend screening appointments appear central to monitoring patients' physical health.

Identified barriers to the successful implementation of tools to facilitate screening can be clustered into resource constraints, environmental barriers, unclear boundaries around professional role and a perceived lack of professional skills and training. Authors in several studies ${ }^{333437-3947}$ noted a number of logistical and resource constraints to the successful collection of measurements due to limited staff time ${ }^{32} 333538$ and difficulty accessing monitoring equipment (such as specific WC tool for obese patients and access to BP monitors in CMHTs). Staff also reported difficulties capturing monitoring results onto the tool ${ }^{28} 323839$ (eg, complicated guidelines to follow). Other barriers included patient resistance to exploring sensitive topics such as sexual health, and transportation, cultural and language barriers to access phlebotomy clinics and arranging an appointment.

Authors in several studies 3233 36-38 47 noted low uptake of physical measurements (eg, WC, fasting BG) and appointment non-adherence to cancer screening and MS monitoring. Lack of integrated care between mental health services and primary care ${ }^{33} 343738$ for treatment and referral postdiagnosis was another barrier. Some mental health staff were reluctant to see MS screening as their responsibility, ${ }^{32}{ }^{33}$ leading to resistance to engage in this activity. The lack of training to collect WC data in a uniform way, and unawareness of a potential 'social desirability bias', ${ }^{28}$ lead to the risk of unreliable results. Lastly, a perceived lack of expertise from mental health professionals to interpret physical health results ${ }^{34}$ was raised as a potential barrier.

Several facilitators to the successful implementation of tools to facilitate screening were identified, including staff feeling invested and having a sense of 'ownership' in physical health monitoring, ${ }^{33} 363739$ staff flexibility around taking measures by using alternative equipment and tests ${ }^{33}$ and having a clinical psychiatric pharma$\operatorname{cist}^{37} 47$ on the ward to support mental health professionals, for example, by reminding staff to request blood tests and provide the relevant guidelines and precautions to follow when hypolipidaemic agents are prescribed.

Barriers to the successful implementation of health service delivery changes are clustered into resource constraints, environmental barriers, unclear boundaries around professional role and patient resistance. Authors note lack of time ${ }^{334047}$ for health professionals to allocate to screening as a barrier, as well as staff turnover ${ }^{4346}$ and other resource constraints ${ }^{41447}$ such as lack of screening equipment and a prescribing provider. Environmental barriers include lack of coordination across the primary and secondary care interface ${ }^{3041445}$ and difficulty for patients and staff to obtain a screening appointment. ${ }^{293}$ Reluctance to engage in screening was observed from the clinician and patient perspective. In staff, limited clarity over who takes responsibility for screening ${ }^{41} 48$ was a barrier, as well as difficulty to engage staff $^{33}{ }^{40}$ in the project, staff resistance to change ${ }^{404546}$ and staff not perceiving physical health screening as a priority. ${ }^{41}{ }^{46} \mathrm{In}$ patients, reluctance to engage with screening was identified as lack of motivation/scepticism in the screening process, ${ }^{41} 46$ appointment non-adherence ${ }^{29} 3342$ and particular resistance to undergo physical measurements. ${ }^{42} 46$

Facilitators to the successful implementation of health service delivery changes include having team 'champions' or a key worker to encourage screening, having staff that feel invested/a sense of ownership with regard to physical health screening, ${ }^{29} 3340-4247$ stakeholder involvement, ${ }^{29} 314042$ having strong links to primary care and specialist services ${ }^{29} 3033424548$ including at-home phlebotomy services and established trust between clients and staff. ${ }^{29}{ }^{334}$ Barriers to the successful implementation of tools and health service delivery changes to facilitate screening include workload issues, resource constraints such as difficulty accessing monitoring equipment, patient resistance to screening and difficulty in arranging an appointment, fragmented links between primary and secondary care, unclear professional role boundaries for screening and staff resistance to engage in screening. Facilitators to the successful implementation of tools and health service delivery changes to facilitate screening include staff feeling invested and a sense of 'ownership' to engage in physical health monitoring. 
The quality of data identified was generally low, it is therefore not possible to determine the size of effect any intervention may have. Different interventions may target different aspects of screening and different barriers and facilitators may apply. However, the high level of heterogeneity and the limited quality of evidence meant that it is not possible to draw firm conclusions. Several potentially useful intervention approaches were identified however. A key aim of this review was to identify what approach worked for whom. However, this was not achieved since few studies tested this. Nevertheless, the review identified specific barriers and facilitators to screening uptake or access in people with SMI which should be considered in future studies.

Future studies should be reported using the Template for Intervention Description and Replication (TIDiER) guidelines $^{52}$ and Medical Research Counci ${ }^{53}$ guidance to make explicit how the components of complex interventions may work. Similarly, use of behaviour change theory was considered in one intervention design ${ }^{43}$ - some studies acknowledged it was not considered-which provides no insight into what might have impacted on staff and service user behaviour to increase uptake. Only few interventions were designed in collaboration with service users, and their preferences were not explored.

There are no longitudinal studies; therefore, this review is unable to clarify if screening is maintained postintervention, and whether the increase in uptake is sustainable or a consequence of the Hawthorne effect whereby health professional behaviour reacts to being observed. An evidence gap was identified as only three studies ${ }^{31} 3246$ reported on whether the results of screening were acted upon, for instance through referral or clinical intervention. One study ${ }^{33}$ aims to make annual MS screening a 'routine responsibility' for the mental health team but acknowledges it cannot refer patients to primary care.

\section{Study limitations}

There is inconsistency around how terms such as 'screening' and 'monitoring' are used which hampers comparative exercises. Our method of deploying them may differ to that of others who may use different terms and include different studies. To compensate for this, and in line with realist review methodology, we used a broad and inclusive study identification process which we adapted iteratively through the study selection process, as described in the Study Selection section. We identified a wide range of studies with varied participants, settings, interventions and intervention targets; a narrower review may provide answers which are more applicable to particular situations; however, the lack of good quality evidence identified suggests that this is unlikely to be the case.

\section{CONCLUSION}

\section{Policy implications}

Interventions to reduce patient and health professional resistance to screening which are informed by behaviour change theory should be developed and tested. Strategies to improve coordination between primary and secondary care are also needed, as are guidelines to clarify professional role boundaries. Resource constraints such as staff time and lack of monitoring equipment in mental health settings need to be addressed in the various clinics where screening occurs. Involving service users in intervention design is also important so that their preferences for location, frequency and type of support can be identified and targeted. Consideration of how interventions are likely to work should be made during development and testing.

Contributors EB, CB, SC and DT were involved in the study design. EB, MGH, FRL-G, AM and DT acquired and analysed the data. EB, SC, FRL-G and PW interpreted the data. FRL-G and AM drafted the manuscript. CB, SC, FRL-G and PW critically revised the manuscript for intellectual content. EB, CB, SC, MG-H, CG, FRL-G, AM, DT and PW contributed to writing and interpretation of the results.

Funding This research received no specific grant from any funding agency in the public, commercial or not-for-profit sectors.

Competing interests None declared.

Patient consent Not required.

Provenance and peer review Not commissioned; externally peer reviewed. Data sharing statement No additional data are available.

Open Access This is an Open Access article distributed in accordance with the Creative Commons Attribution Non Commercial (CC BY-NC 4.0) license, which permits others to distribute, remix, adapt, build upon this work non-commercially, and license their derivative works on different terms, provided the original work is properly cited and the use is non-commercial. See: http://creativecommons.org/ licenses/by-nc/4.0/

(c) Article author(s) (or their employer(s) unless otherwise stated in the text of the article) 2018. All rights reserved. No commercial use is permitted unless otherwise expressly granted.

\section{REFERENCES}

1. Walker ER, McGee RE, Druss BG. Mortality in mental disorders and global disease burden implications: a systematic review and metaanalysis. JAMA Psychiatry 2015;72:334-41.

2. DE Hert M, Correll CU, Bobes J, et al. Physical illness in patients with severe mental disorders. I. Prevalence, impact of medications and disparities in health care. World Psychiatry 2011;10:52-77.

3. Chang CK, Hayes RD, Perera G, et al. Life expectancy at birth for people with serious mental illness and other major disorders from a secondary mental health care case register in London. PLoS One 2011;6:e19590.

4. Brown S, Kim M, Mitchell C, et al. Twenty-five year mortality of a community cohort with schizophrenia. Br J Psychiatry 2010;196:116-21.

5. Kisely S, Crowe E, Lawrence D. Cancer-related mortality in people with mental illness. JAMA Psychiatry 2013;70:209-17.

6. Howard LM, Barley EA, Davies E, et al. Cancer diagnosis in people with severe mental illness: practical and ethical issues. Lancet Oncol 2010;11:797-804.

7. Cunningham R, Sarfati D, Stanley J, et al. Cancer survival in the context of mental illness: a national cohort study. Gen Hosp Psychiatry 2015;37:501-6.

8. Holt RI, Mitchell AJ. Diabetes mellitus and severe mental illness: mechanisms and clinical implications. Nat Rev Endocrinol 2015;11:79-89.

9. Mulligan K, McBain H, Lamontagne-Godwin F, et al. Barriers and enablers of type 2 diabetes self-management in people with severe mental illness. Health Expect 2017;20:1020-30.

10. World Health Organization - Department of Noncommunicable Disease Surveillance. Definition, diagnosis and classification of diabetes mellitus and its complications: report of a WHO consultation. Part 1, diagnosis and classification of diabetes mellitus. Geneva, Switzerland: World Health Organization, 1999. WHO/NCD/ NCS/99.2. 
11. Royal College of Psychiatrists. Report of the Second Round of the National Audit of Schizophrenia (NAS). London: Healthcare Quality Improvement Partnership, 2014.

12. Woodhead $\mathrm{C}$, Cunningham R, Ashworth $\mathrm{M}$, et al. Cervical and breast cancer screening uptake among women with serious mental illness: a data linkage study. BMC Cancer 2016;16:819.

13. Aggarwal A, Pandurangi A, Smith W. Disparities in breast and cervical cancer screening in women with mental illness: a systematic literature review. Am J Prev Med 2013;44:392-8.

14. Weinstein LC, LaNoue M, Hurley K, et al. Using concept mapping to explore barriers and facilitators to breast cancer screening in formerly homeless women with serious mental illness. J Health Care Poor Underserved 2015;26:908-25.

15. Mitchell AJ, Pereira IE, Yadegarfar M, et al. Breast cancer screening in women with mental illness: comparative meta-analysis of mammography uptake. Br J Psychiatry 2014;205:428-35.

16. Camilloni L, Ferroni E, Cendales BJ, et al. Methods to increase participation in organised screening programs: a systematic review. BMC Public Health 2013;13:464.

17. Barley EA, Borschmann RD, Walters P, et al. Interventions to encourage uptake of cancer screening for people with severe mental illness. Cochrane Database Syst Rev 2016;9. Issue9.Art. No:CD009641.

18. Clifton A, Burgess C, Clement S, et al. Influences on uptake of cancer screening in mental health service users: a qualitative study. BMC Health Serv Res 2016;16:257.

19. Pawson R, Greenhalgh T, Harvey G, et al. Realist review--a new method of systematic review designed for complex policy interventions. J Health Serv Res Policy 2005;10(suppl 1):21-34.

20. Holland C, Cooper Y, Shaw R, et al. Effectiveness and uptake of screening programmes for coronary heart disease and diabetes: a realist review of design components used in interventions. BMJ Open 2013;3:e003428

21. Wong G, Westhorp G, Manzano A, et al. RAMESES II reporting standards for realist evaluations. BMC Med 2016;14:96.

22. Public Health England. NHS population screening explained. Population screening programmes - Screening and quality assurance. 2. London: Public Health England, 2013.

23. Tosh G, Clifton AV, Xia J, et al. Physical health care monitoring for people with serious mental illness. Cochrane Database Syst Rev 2014:CD008298. Issue 1.

24. Barley E, Burgess C, Clement S, et al. Uptake of physical health screening in people with severe mental illness: a realist review. PROSPERO 2016. CRD42016047848.

25. Ferrara M, Mungai F, Miselli M, et al. Strategies to implement physical health monitoring in people affected by severe mental illness: a literature review and introduction to the Italian adaptation of the Positive Cardiometabolic Health Algorithm. Psychopathology 2015;21:269-80.

26. Hardy S, Gray R. Is the use of an invitation letter effective in prompting patients with severe mental illness to attend a primary care physical health check? Prim Health Care Res Dev 2012;13:347-52.

27. Hardy S, Deane K, Gray R. The Northampton Physical Health and Wellbeing Project: the views of patients with severe mental illness about their physical health check. Ment Health Fam Med 2012;9:233-40.

28. Bressington D, Mui J, Hulbert S, et al. Enhanced physical health screening for people with severe mental illness in Hong Kong: results from a one-year prospective case series study. BMC Psychiatry 2014;14:57.

29. Heyding RK, Cheung AM, Mocarski EJ, et al. A communitybased intervention to increase screening mammography among disadvantaged women at an inner-city drop-in center. Women Health 2005:41:21-31.

30. Xiong GL, Bermudes RA, Torres SN, et al. Use of cancer-screening services among persons with serious mental illness in Sacramento County. Psychiatr Serv 2008;59:929-32.

31. Millar HL. Development of a health screening clinic. Eur Psychiatry 2010;25 Suppl 2:S29-S33.

32. Kioko E, Williams K, Newhouse B. Improving metabolic syndrome screening on patients on second generation antipsychotic medication. Arch Psychiatr Nurs 2016;30:671-7.
33. Castillo EG, Rosati J, Williams C, et al. Metabolic syndrome screening and assertive community treatment: a quality improvement study. J Am Psychiatr Nurses Assoc 2015;21:233-43.

34. Gonzalez C, Ahammed N, Fisher R. Improving physical health monitoring for out-patients on antipsychotic medication. Psychiatrist 2010;34:91-4.

35. Hardy S, Hinks P, Gray R. Does training practice nurses to carry out physical health checks for people with severe mental illness increase the level of screening for cardiovascular risk? Int J Soc Psychiatry 2014;60:236-42.

36. Shuel $\mathrm{F}$, White J, Jones $\mathrm{M}$, et al. Using the serious mental illness health improvement profile [HIP] to identify physical problems in a cohort of community patients: a pragmatic case series evaluation. Int J Nurs Stud 2010;47:136-45.

37. Vasudev K, Thakkar PB, Mitcheson N. Physical health of patients with severe mental illness: an intervention on medium secure forensic unit. Int J Health Care Qual Assur 2012;25:363-70.

38. Wiechers IR, Viron M, Stoklosa J, et al. Impact of a metabolic screening bundle on rates of screening for metabolic syndrome in a psychiatry resident outpatient clinic. Acad Psychiatry 2012;36:118-21.

39. Yeomans D, Dale K, Beedle K. Systematic computerised cardiovascular health screening for people with severe mental illness. Psychiatr Bull 2014;38:280-4.

40. Abdallah N, Conn R, Latif Marini A, et al. Improving physical health monitoring for patients with chronic mental health problems who receive antipsychotic medications. BMJ Qual Improv Rep 2016;5:u210300.w4189.

41. Latoo J, Omodunbi O, Hindley D, et al. Physical health of people with severe mental illness: Don't just screen... intervene!. Br J Med Pract 2015;8:a821.

42. Vasudev K, Martindale BV. Physical healthcare of people with severe mental illness: everybody's business!. Ment Health Fam Med 2010;7:115-22.

43. Thompson A, Hetrick SE, Alvarez-Jiménez M, et al. Targeted intervention to improve monitoring of antipsychotic-induced weight gain and metabolic disturbance in first episode psychosis. Aust $N Z \mathrm{~J}$ Psychiatry 2011;45:740-8.

44. Druss BG, von Esenwein SA, Compton MT, et al. A randomized trial of medical care management for community mental health settings: the Primary Care Access, Referral, and Evaluation (PCARE) study. Am J Psychiatry 2010;167:151-9.

45. Osborn DP, Nazareth I, Wright CA, et al. Impact of a nurse-led intervention to improve screening for cardiovascular risk factors in people with severe mental illnesses. Phase-two cluster randomised feasibility trial of community mental health teams. BMC Health Serv Res 2010;10:61.

46. Rosenbaum S, Nijjar S, Watkins A, et al. Nurse-assessed metabolic monitoring: a file audit of risk factor prevalence and impact of an intervention to enhance measurement of waist circumference. Int $J$ Ment Health Nurs 2014;23:252-6.

47. DelMonte MT, Bostwick JR, Bess JD, et al. Evaluation of a computerbased intervention to enhance metabolic monitoring in psychiatry inpatients treated with second-generation antipsychotics. J Clin Pharm Ther 2012;37:668-73.

48. Wilson E, Randall C, Patterson S, et al. Monitoring and management of metabolic abnormalities: mixed-method evaluation of a successful intervention. Australas Psychiatry 2014;22:248-53.

49. Higgins JP, Altman DG, Gøtzsche PC, et al. The Cochrane Collaboration's tool for assessing risk of bias in randomised trials. BMJ 2011;343:d5928.

50. von Elm E, Altman DG, Egger M, et al. The Strengthening the Reporting of Observational Studies in Epidemiology (STROBE) statement: guidelines for reporting observational studies. Ann Intern Med 2007;147:573-7.

51. Sanderson S, Tatt ID, Higgins JP. Tools for assessing quality and susceptibility to bias in observational studies in epidemiology: a systematic review and annotated bibliography. Int J Epidemiol 2007;36:666-76.

52. Armstrong R, Campbell M, Craig P, et al. Reporting guidelines for population health and policy interventions: TIDieR-PHP. The Lancet 2015;386(Supp 2):S19

53. Anderson R. New MRC guidance on evaluating complex interventions. BMJ 2008;337:a1937. 